\title{
BUNCH BY BUNCH FEEDBACK SYSTEMS FOR THE KEKB RINGS
}

\author{
M. Tobiyama, E. Kikutani, J. W. Flanagan and S. Hiramatsu \\ Accelerator Laboratory, High Energy Accelerator Research Organization (KEK), \\ 1-1 Oho, Tsukuba, Ibaraki 305-0801, Japan
}

\begin{abstract}
Transverse bunch-by-bunch feedback systems for damping coupled-bunch instabilities in the KEKB rings have been working since the early stages of the commissioning of the rings. Stabilization of the beams with the system has been successful, permitting tests of higher beam currents and various filling patterns. The performance of these systems has been demonstrated under the normal physics run pattern of $8 \mathrm{~ns}$ bunch spacing over long-term operation, and also under special filling patterns with the minimum bunch spacing of $2 \mathrm{~ns}$.
\end{abstract}

\section{INTRODUCTION}

The KEKB collider, which consists of an $8 \mathrm{GeV}$ electron ring (HER) and a $3.5 \mathrm{GeV}$ positron ring (LER), is designed to accumulate very high beam currents with many bunches. About 5000 bunches per ring will be stored at total beam currents of 1.1 A (HER) and 2.6 A (LER) in the design goal. The commissioning of the rings started at the end of 1998 and is continuing up to now. Even at early stages of the commissioning, we have encountered unexpectedly strong transverse coupled-bunch instabilities in both rings which limited the total storable currents to very low values, about $50 \mathrm{~mA}$. With the progress of the operation of the transverse bunch-by-bunch feedback systems, we have successfully suppressed the instabilities and have been extending the total currents. We can store over $940 \mathrm{~mA}$ in the LER, $775 \mathrm{~mA}$ in the HER without coherent beam oscillation and have achieved a peak luminosity of $4.04 \times 10^{33} \mathrm{~cm}^{-2} \mathrm{~s}^{-1}$. The bunch feedback system consists of position detection systems, high-speed digital signal processing systems with a base clock of $509 \mathrm{MHz}$, and wide-band kickers fed by wide-band, high-power amplifiers. We describe here the progress and the present status of our feedback systems. Also the related beam diagnostic tools such as the large scale memory board will be shown.

\section{OUTLINE OF KEKB BUNCH FEEDBACK SYSTEMS}

All of the feedback equipment is installed in the Fuji crossing area as shown in Fig. 1. There are two sections of position monitors in each ring to make a suitable betatron phase shift to the transverse kicker. Stripline-type kickers for transverse deflection are installed upstream of the first monitor chamber. We use two kinds of transverse kickers, a $40 \mathrm{~cm}$ wideband kicker up to $255 \mathrm{MHz}$ and a $1.2 \mathrm{~m}$ lower frequency kicker for frequencies below $1 \mathrm{MHz}$. We have also installed two of the DA $\Phi$ NE type longitudinal kickers[1] in the LER, each with four input ports and four output ports.

A block diagram of the transverse feedback system is shown in Fig. 2. The major part of closed orbit distortion

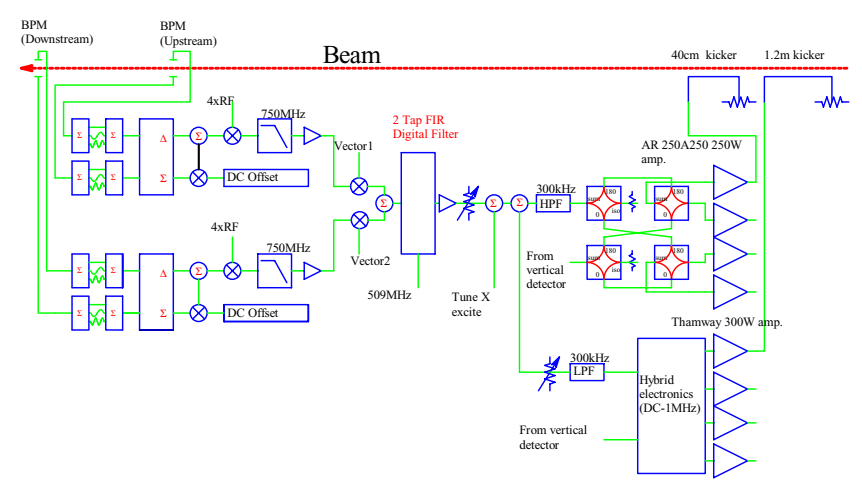

Figure 2: Block diagram of the transverse feedback systems.

(COD) at the BPMs is suppressed by continuous closedorbit correction (CCC)[2]. Residual CODs are cancelled by the local offset canceller circuit. The phase advance and the betatron function at the monitors and the kickers are listed in Table 1. We adjust the phase difference between

Table 1: Feedback related parameters of KEKB.

\begin{tabular}{lcl}
\hline Ring & LER/HER & \\
\hline Energy & $3.5 / 8.0$ & $\mathrm{GeV}$ \\
Circumference & 3016.26 & $\mathrm{~m}$ \\
Bunch current & $0.76 / 0.67$ & $\mathrm{~mA}$ \\
Betatron tune & $45.53 / 43.58(\mathrm{LER})$ & \\
& $44.52 / 41.62(\mathrm{HER})$ & \\
RF voltage & $6.0 / 11.0$ & $\mathrm{MV}$ \\
RF Frequency & 508.887 & $\mathrm{MHz}$ \\
Damping time (L) & $22 / 23$ & $\mathrm{~ms}$ \\
\hline Betatron functions & $\beta_{x} / \beta_{y}$ & \\
BPM1 & $21 \mathrm{~m} / 21 \mathrm{~m}$ & LER \\
& $19 \mathrm{~m} / 16 \mathrm{~m}$ & HER \\
\multicolumn{1}{c}{ BPM2 } & $21 \mathrm{~m} / 21 \mathrm{~m}$ & LER \\
& $30 \mathrm{~m} / 8 \mathrm{~m}$ & HER \\
\multicolumn{1}{c}{ Kickers } & $23 \mathrm{~m} / 7 \mathrm{~m}$ & LER \\
& $33 \mathrm{~m} / 14 \mathrm{~m}$ & HER \\
Phase advance & $\Delta \nu_{x} / \Delta \nu_{y}$ & \\
BPM1 - BPM2 & $65^{\circ} / 65^{\circ}$ & LER \\
& $88^{\circ} / 107^{\circ}$ & HER \\
\multicolumn{1}{c}{ Kicker - BPM1 } & $18^{\circ} / 48^{\circ}$ & LER \\
& $10^{\circ} / 12^{\circ}$ & HER \\
\hline
\end{tabular}




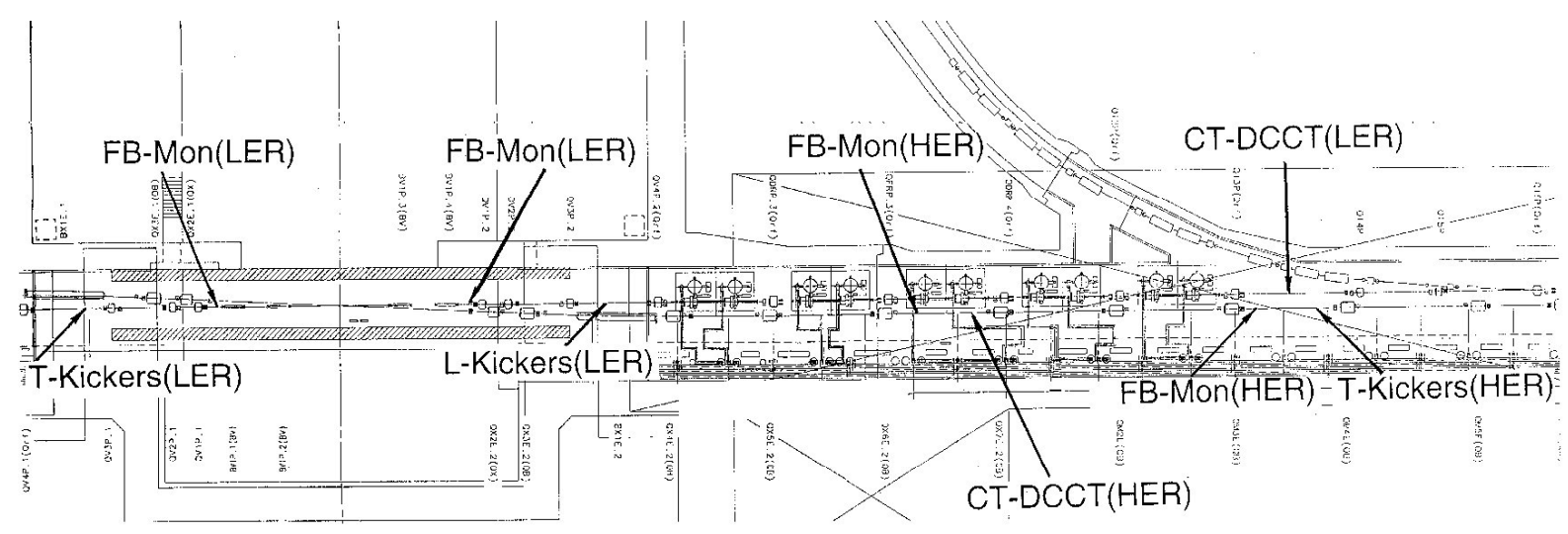

Figure 1: Location of the feedback equipment at the Fuji crossing area. Positrons come from left side and electrons come from the right side. All the final feedback amplifiers are installed under the crossing bridge.

the monitor and the kickers by vectorially combining the two signals from the upstream and downstream positions. The signal processing systems[3] are working as two-tap FIR digital filters. The function of the two-tap FIR filter are (1) rejection of DC component including error in the detection circuit, (2) enhance the betatron tune component, and (3) digital delay to adjust the one-turn delay. The tap positions of the filters are set with (kick) $\propto$ (position data 2 turns before ) - (position data 1 turn before) because the fractional part of the betatron tunes are around 0.5. The residual phase errors coming from the phase shift in the filter function are corrected with fine tuning of the vectorially combining circuits.

Four $250 \mathrm{~W}$ amplifiers $(10 \mathrm{kHz} \sim 250 \mathrm{MHz})$ to drive the $40 \mathrm{~cm}$ kicker, and four $300 \mathrm{~W}$ amplifiers $(5 \mathrm{kHz} \sim 1 \mathrm{MHz})$ to drive the $1.2 \mathrm{~m}$ kicker, are used for each ring. Since the performance of the wideband amplifiers below $50 \mathrm{kHz}$ is not ideal, and intentional injection error (kicker jump) has only lower frequency components, we use the lower frequency system to help the damping around the lowest mode. To equalize the two bands, we use 1 st order low pass and high pass filter with a crossover frequency of $300 \mathrm{kHz}$.

Though we have prepared a wideband longitudinal feedback system, we have encountered no longitudinal instability except for an RF-noise induced one. We have, therefore, not used them up to now.

\section{EXPERIENCE WITH THE FEEDBACK SYSTEMS}

In the early stage of the commissioning of the feedback systems[4], we operated the systems with a very simplified scheme because both the feedback systems and the accelerator were not yet well understood. We used only the wideband kicker systems to avoid the complexity concerning the equalization of overlapping bands. The digital filter worked as a simple digital delay, not as a two-tap filter. With this mode of operation, we had several difficulties:

- Unexpected power saturation at the final power am- plifiers. As the residual offset of a detector depends on both the bunch current and beam phase shift due to beam loading, it is impossible to cancel out the offset with the analog circuit. The saturation reduced the dynamic range of the feedback system and restricted the gain of the system to be lower than expected.

- Longer damping time at injection. As the shunt impedance and the maximum power of the wideband system is limited, the amplifiers always saturate at the injection period due to injection bump error. The system worked as the bang-bang damping scheme during injection and the performance was reduced greatly.

By changing the function of the digital filter from the simple delay mode to 2 tap FIR mode, position offsets between the bunches has been completely cancelled out. We have increased the feedback gain without saturation at the amplifiers. The huge perturbation during injection is now suppressed well by adding the lower frequency system and equalizing the wideband system, though the gain balances between the two systems are still problematic.

We have measured the damping rate of the horizontal oscillation during injection using the bunch oscillation recorder (BOR)[3]. Figure 3 shows an example of damping of horizontal oscillation during injection of HER with total current around $730 \mathrm{~mA}$. The present setting of the feedback damping time at maximum beam current is $0.2 \mathrm{~ms}$ (horizontal:H) and $0.8 \mathrm{~ms}$ (vertical:V) in HER, $0.2 \mathrm{~ms}(\mathrm{H})$ and $0.4 \mathrm{~ms}(\mathrm{~V})$ in LER.

In normal operation for physics running, we fill the beam every 4 th bucket ( $8 \mathrm{~ns}$ spacing) with a gap space for the beam abort kicker, with a total bunch number of 1155 per ring. Under this fill pattern mode, the growth time of the instabilities in the LER are less than $0.5 \mathrm{~ms}$ in horizontal, and about a few ms in vertical. In the HER, the growth time is about a few ms for both horizontal and vertical planes. For machine development, we have also tried several special filling patterns, such as $(11001100 \cdots),(101010 \cdots)$ and $(100100 \cdots)$, where 1 means a filled bucket and 0 means 


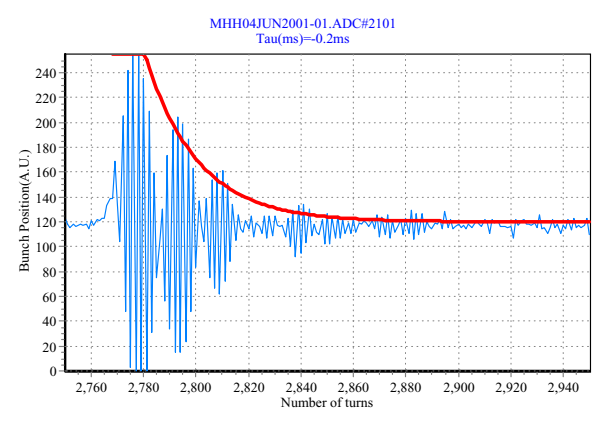

Figure 3: Feedback damping of injection oscillation (horizontal) in the HER at current of $730 \mathrm{~mA}$.

an empty bucket. Though the growth rate of the instability for several patterns are comparable to or higher than the feedback damping time and so cannot easily handle higher beam currents, the feedback system has shown much better performance than design. Figure 4 shows an example of a special filling pattern (4 ns spacing), before and after switching off/on the horizontal feedback system. By

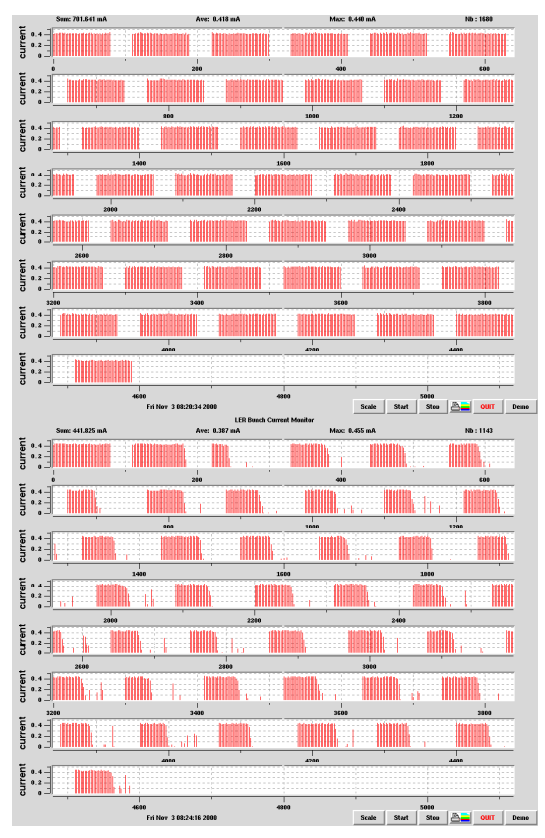

Figure 4: Special filling pattern before (upper) and after (lower) switching off/on the feedback.

switching off the horizontal feedback system for $41 \mathrm{~ms}$, the beam current is reduced from $650 \mathrm{~mA}$ to $390 \mathrm{~mA}$ within a few ms and the tails of the bunch trains were lost. The analysis of this growth by using the BOR shows very quick growth, less than $0.5 \mathrm{~ms}$, and some broad modes around the lowest modes.

The growth rates of the instabilities for both rings at the normal filling pattern of $8 \mathrm{~ns}$ spacing are much faster than the ones expected in the design stage. Candidates for such instability sources are photo-electron induced instability in the LER and fast beam-ion instability in the HER, but these are not confirmed yet. To investigate the cause of sudden beam loss during operation, we are now testing the beam loss trigger systems to record the beam behavior just before the beam loss with BORs. An example of the growth of vertical oscillation just before beam loss in the HER is shown in Fig. 5.

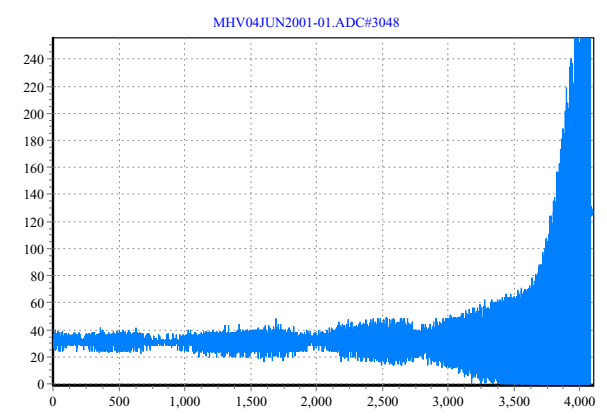

Figure 5: Growth of vertical oscillation just before beam loss in the HER.

\section{SUMMARY}

The transverse bunch-by-bunch feedback systems for KEKB rings have been contributing to both the commissioning of the rings and the operation of the colliding experiment from early stages of the commissioning up to present, about 2.5 years of operation. A damping time of around $0.2 \mathrm{~ms}$ (20 turns) has been achieved under stable operation of the feedback systems.

To increase the luminosity, we will continue to try to increase both the beam current and the total number of bunches. Experiments of special filling patterns suggest the existence of large impedance source in the ring. The feedback systems and the analysis tools such as the BOR with transient-domain analysis or the beam loss trigger will continue to play important roles.

The authors would like to express their sincere appreciation to Professor S. Kurokawa for his continued help and useful suggestions. They also wishes to express their gratitude to Prof. T. Kasuga, Dr. T. Obina, and Dr. Y. Ohnishi for numerous thoughtful discussions. The authors are grateful to the commissioning group of KEKB.

\section{REFERENCES}

[1] R. Boni et al., Particle Accelerators, 52(1996)96.

[2] H. Koiso, private communication.

[3] M. Tobiyama and E. Kikutani, Phys. Rev. ST Accl. Beams 3,012801(2000).

[4] M. Tobiyama et. al., in Proceedings of 12th Symp. Accel. Sci. and Tech, Wako, Saitama, Japan (1999) p.555. 